\title{
Comportement dynamique des lignes d'arbre de turbomachines
}

\author{
Dynamical behavior of turbomachines shafting
}

\author{
par O. Bonneau \\ Laboratoire de Mécanique des Solides, Université de Poitiers \\ G. Jacquet \\ Laboratoire de Mécaniques des Structures, INSA de Lyon \\ S. Berger \\ Laboratoire de Modélisation Intelligence Processus Systèmes \\ Automatique, Ecole Supérieure des Sciences Appliquées de Mulhouse (ESSAIM).
}

High speed rotors set a lot of stability problems especially when the speed of rotation is going through a critical speed. The dynamical behavior of the fluid bearings has, in that case, an important effect. This study presents two examples of coupling between fluid element and flexibility of the shaft:

- the coupling between the axial thrust bearing behavior and the bending vibrations of the shaft will be studied.

- the behavior of a shaft with flexible wheel-shaft and bearing-shaft coupling.

\section{INTRODUCTION}

Les contraintes économiques de l'industrie poussent les fabricants à augmenter l'ensemble des capacités de leurs machines. Le domaine de la machine tournante n'échappe pas à la règle, les constructeurs sont obligés de faire appel à des modélisations de plus en plus fines afin de déplacer les limites de fonctionnement. De nombreuses disciplines sont concernées par ces études : la dynamique des structures, l'acoustique, le traitement du signal, la mécanique des fluides, la métallurgie, l'aérodynamique, la thermique, la lubrification... La difficulté mais aussi la richesse de l'étude des rotors tient à ces couplages et au choix des différents modèles qui devront être employés. Jusqu'à ces dernières années, chaque discipline développait ses propres applications tout en simplifiant les modèles relevant des autres disciplines. Cette démarche conduit à des résultats qui peuvent être satisfaisants pour une étude d'avant-projet, mais elle reste très limitée lorsque les mécanismes fonctionnent dans des conditions sévères, au voisinage des vitesses critiques par exemple. Il est maintenant indispensable dans de nombreuses études de réaliser un couplage fluide-structure, en raison notamment des fortes non-linéarités pouvant apparaître dans certaines applications.
Nous présenterons dans un premier temps les éléments techniques faisant partie de l'environnement du rotor : l'environnement fluide (turbine, corps de pompe), les supports de ligne d'arbre (paliers, roulements) et l'étanchéité (joint lisse, labyrinthe...). Puis nous détaillerons dans un deuxième temps deux études : une étude sur l'influence d'une butée fluide axiale avec défaut sur le comportement non linéaire d'un arbre flexible monté sur éléments fluides et une étude du comportement couplé roue-arbre-fluide réalisée à partir d'une modélisation tri-dimensionnelle.

\subsection{L'environnement fluide}

Le comportement dynamique des rotors et en particulier des turbomachines est en grande partie conditionné par le fluide en présence. Nous présenterons dans ce paragraphe les principaux fluides rencontrés dans nos études.

\subsubsection{L'huile}

Les nombres de Reynolds engendrés par une lubrification à l'huile sont généralement assez faibles. L'écoulement reste donc principalement laminaire avec des effets d'inertie négligeables vis-à-vis des effets visqueux. L'huile est considérée comme incompressible. C'est ce type de lubrifiant qui est 
employé en lubrification "classique" au niveau des paliers, butées, film amortisseur... Les principaux problèmes apparaissent au niveau de la modélisation de l'élément fluide luimême, le fluide ne posant pas de problème particulier.

\subsubsection{L'eau}

L'eau est utilisée dans de nombreuses installations industrielles. Les turbopompes, par exemple, fournissent à l'eau une énergie cinétique et un gradient de pression importants. La ligne d'arbre est en général montée sur des paliers à roulements ou sur des paliers hydrodynamiques à patins oscillants, des joints annulaires de type labyrinthes réalisant l'étanchéité partielle entre les différentes zones de pression. La faible viscosité de l'eau, les grandes vitesses de rotation, les jeux importants et les gradients de pression conduisent à un écoulement très turbulent. $[1,2]$. Le travail essentiel est alors de connaître au mieux l'effet de cette turbulence sur le comportement dynamique des éléments fluides. De plus les effets d'inertie du fluide ne sont plus négligeables et doivent être intégrés dans les modélisations [3]. Par ailleurs les problèmes que nous avons été amenés à traiter nous ont conduit à envisager le comportement d'un fluide tel que l'hydrogène liquide.

Le comportement dynamique d'une structure en fonctionnement dans un fluide dense est sensiblement différent du comportement de la même structure en air $[4,5]$. Le fluide apporte de la masse ajoutée qui entraîne une baisse de fréquence ainsi que de l'amortissement qui diminue les niveaux vibratoires. Les modes propres de vibration identifiés en air se retrouvent en eau mais l'immersion a pour effet de faire apparaître de nouveaux modes : par exemple pour les roues de turbomachines les modes se dédoublent et les modes résultants se distinguent par des flasques de roues qui vibrent en phase et en opposition de phase.

\subsubsection{L'air}

Les problèmes rencontrés sur les turbocompresseurs sont essentiellement des problèmes liés à l'aérodynamique. Par exemple, l'instabilité de flottement est issue d'un couplage qui se crée entre la vibration d'un aubage et le fluide qui le traverse $[5,6,7]$. Le couplage entre ces phénomènes, la dynamique des rotors et les équations de la lubrification font l'objet de quelques études mais ceci reste un domaine très difficile à explorer.

\subsubsection{Les fluides électrorhéologiques}

Ces fluides, constitués d'une huile dans laquelle des additifs polarisés sont incorporés, ont la propriété d'évoluer lorsque qu'un champ électrique leur est appliqué. Sous l'effet d'un champ électrique, le comportement du fluide est proche de celui d'un fluide de Bingham (les graisses par exemple) mais avec des propriétés qui peuvent être ajustées. L'idée de notre travail est de constituer des éléments de support d'arbre ayant des propriétés adaptatives et ceci afin de pouvoir envisager un contrôle actif du comportement de l'arbre [8,9].

\subsection{Les supports de ligne d'arbre}

Les lignes d'arbres sont supportées en général par deux types d'éléments : les paliers à roulements et les paliers fluides qui seront plus spécialement étudiés ici.

- Les roulements ont un comportement qui peut être considéré comme assez linéaire avec une prédominance de la raideur par rapport à un faible amortissement.
- Les paliers fluides ont un comportement complexe, une première approche linéaire peut être réalisée sous certaines conditions. Il existe cependant de nombreuses applications où une modélisation non linéaire est indispensable.

Nous avons étudié l'influence de deux familles de supports d'arbre : le palier traditionnel et le film amortisseur (squeeze film damper) sur la dynamique du rotor. Le palier traditionnel possède des propriétés de raideur et d'amortissement [10, 11]. Le film amortisseur est un composant qui va privilégier le caractère amortisseur, ce qui va permettre, par exemple, de passer avec succès les différentes vitesses critiques d'un rotor très flexible [12].

Par ailleurs, de nouveaux supports apparaissent tels que les paliers magnétiques qui sont utilisés sur des lignes d'arbres très spécifiques (certains turbocompresseurs par exemple ou pour supporter des broches de machines-outils en usinage très haute vitesse). Cette technologie permet de contrôler en temps réel les caractéristiques du palier.

\subsection{Les éléments d'étanchéité}

Les éléments d'étanchéité vont eux aussi conditionner le comportement dynamique général de la ligne d'arbre. Deux types de joint d'étanchéité sont étudiés au Laboratoire de Mécanique des Solides : les joints à face radiale et les joints annulaires et labyrinthes.

Les caractéristiques des joints à face radiale, étudiés en particulier par B. Tournerie $[13,14,15,16]$, peuvent être intégrés sous forme linéaire dans un calcul de ligne d'arbre et pourront à terme être introduites sous forme non linéaire.

Les joints annulaires et labyrinthes sont modélisés d'un point de vue dynamique par la théorie linéaire en intégrant les effets non linéaires de la turbulence et d'inertie du fluide $[1,2,3,17,18]$. Le calcul dynamique non linéaire impliquerait des temps de calcul très importants.

\subsection{Bilan}

Les descriptifs précédents mettent en évidence la complexité et la diversité des éléments constitutifs d'une ligne d'arbre. Le rotor doit être étudié dans sa globalité : le rotor, les supports de rotor, le fluide, les étanchéités. De nombreuses équipes développent des domaines particuliers mais il est très important d'effectuer des études "horizontales" intégrant simultanément les compétences des différents partenaires. C'est pourquoi le Laboratoire de Mécanique des Solides de l'Université de Poitiers s'est associé au Laboratoire de Mécanique des Structures de l'INSA de Lyon afin de mettre en place dès 1989 un modèle complet de ligne d'arbre souple monté sur des films amortisseurs ayant un comportement fortement non linéaire $[11,12,19]$.

\section{II $\square$ COUPLAGE PAR BUTÉE FLUIDE DES VIBRATIONS AXIALES D'UN ARBRE ET DES VIBRATIONS DE FLEXION}

Nous avons effectué depuis quelques années différentes études permettant de mettre en évidence le couplage entre le comportement d'une butée axiale et la réponse dynamique en flexion de l'arbre. Une étude sur l'influence des défauts de fabrication (sur la butée) a mis en évidence l'apparition d'une perturbation dynamique synchrone $[20,21]$. Seule l'étude non linéaire a pu permettre d'identifier ce phénomène.

Nous allons dans ce paragraphe mettre en évidence le cou- 
plage (par l'élément fluide) du comportement axial avec les vibrations de flexion.

\subsection{La butée}

La butée hydrodynamique est une butée à quatre patins fixes inclinés. La figure 1 présente le paramétrage de la butée, $\left(\mathrm{o}_{0}, \mathrm{x}_{0}, \mathrm{y}_{0}, \mathrm{z}_{0}\right)$ est le repère de référence, $\left(\mathrm{o}_{1}, \mathrm{x}_{1}, \mathrm{y}_{1}, \mathrm{z}_{1}\right)$ et $\left(\mathrm{o}_{2}, \mathrm{x}_{2}, \mathrm{y}_{2}, \mathrm{z}_{2}\right)$ sont respectivement liés au stator et au rotor. L'axe $z_{2}$ est l'axe de rotation du rotor. Les mouvements du rotor (au niveau du centre $\mathrm{O}_{2}$ de la butée) sont limités aux trois translations et aux deux rotations autour de $x_{0}$ et $y_{0}$. La résolution de l'équation des films minces visqueux (sur chaque patin) est effectuée par différences finies.

Les forces et moments du fluide sur l'arbre sont obtenus par intégration du champ de pression sur la surface du rotor.

L'arbre est discrétisé par éléments finis de type rotor, une réduction modale est effectuée de façon à diminuer le nombre de degrés de liberté $[11,12]$.

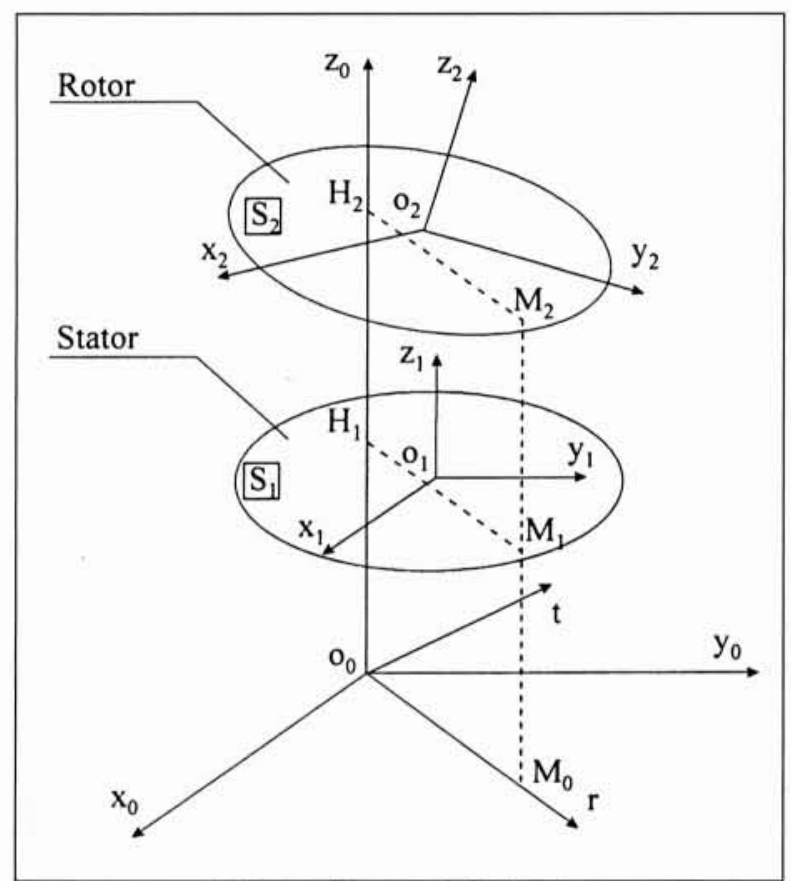

1. Paramétrage de la butée.

La simulation dynamique non linéaire de l'arbre monté sur support fluide est un calcul itératif dans le temps (pas à pas). A chaque pas de temps les champs de pression au sein des différents supports fluides sont calculés (en fonction des vitesses et positions). Cela permet d'obtenir l'ensemble des actions mécaniques sur l'arbre. Un passage dans la base modale permet alors de calculer les accélérations modales qui, une fois intégrées (par une méthode de type Euler à pas variable), donneront les nouvelles vitesses et positions modales. Les deux supports fluides ont un comportement qui sera modélisé par la théorie du palier court. Le modèle utilisé permet de tenir compte de leur non linéarité (même si ici ils ont un comportement assez linéaire).

\subsection{Application}

Le rotor est monté dans deux paliers identiques et une butée assure la reprise des efforts axiaux. Une charge statique et dynamique axiale est exercée sur l'arbre ainsi qu'une légère charge radiale.

Le rotor est monté dans deux paliers identiques et une butée assure la reprise des efforts axiaux. La figure 2 présente la géométrie de l'arbre. Une excitation statique et dynamique (à fréquence variable) axiale est appliquée sur l'arbre.

L'arbre est excité dans la direction axiale par une charge de forme sinusoïdale, dont la fréquence augmente linéairement de $0 \mathrm{~Hz}$ à $600 \mathrm{~Hz}$ en $24 \mathrm{~s}$. La vitesse de rotation de l'arbre est fixée à $10000 \mathrm{tr} / \mathrm{min}$.

\subsection{Résultats}

Le comportement dynamique linéaire de cet arbre a été effectué $[20,21]$; il présente un premier mode de flexion autour de $12400 \mathrm{tr} / \mathrm{min}$. Les amplitudes des trajectoires de l'arbre sont présentées dans les figures 3 et 4 .

La figure 3 présente l'amplitude du milieu de l'arbre (ventre de vibration) et la figure 4 , les amplitudes au droit des paliers. Ces amplitudes sont adimensionnées par le jeu des paliers $(30 \mu \mathrm{m})$.

Au milieu de l'arbre, il apparaît un pic de résonance lorsque la charge axiale excite l'arbre avec une fréquence égale à $210 \mathrm{~Hz}(12600 \mathrm{tr} / \mathrm{mn})$. Cette fréquence de résonance apparaît également dans l'amplitude des trajectoires dans les paliers. Un second pic plus petit survient principalement dans le palier 2 à une fréquence d'environ $90 \mathrm{~Hz}$, ce qui correspond à un mode de paliers.

\subsection{Conclusion}

Les résultats présentés ci-dessus mettent en évidence le couplage qui peut exister entre les vibrations axiales de la ligne d'arbre et ses réponses en flexion. Nous avons par ailleurs $[16,17]$ montré qu'un défaut de réalisation de la butée pouvait venir exciter un mode de flexion de l'arbre. Une étude classique, linéaire, n'aurait pas pu mettre en évidence ces phénomènes.

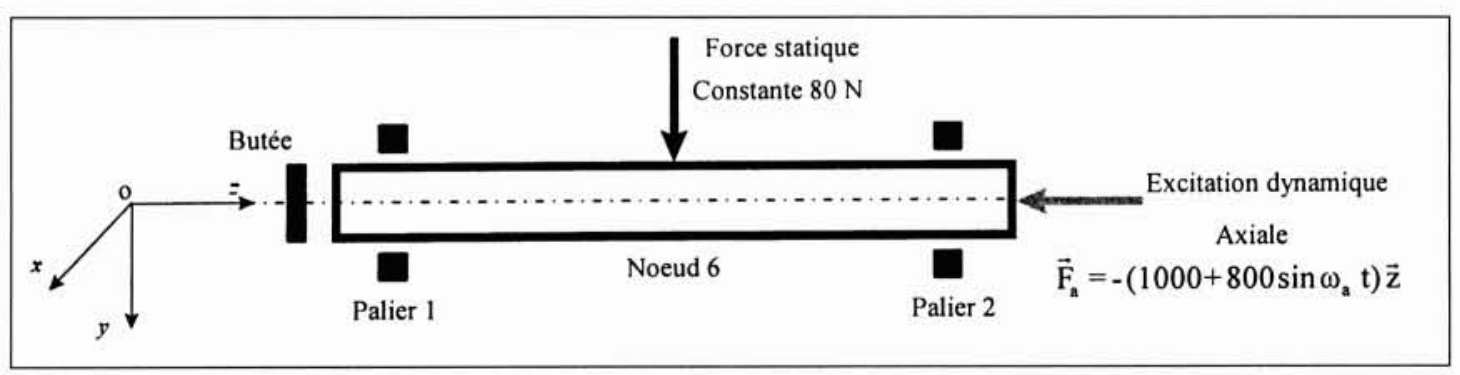

2. Système tournant étudié. 


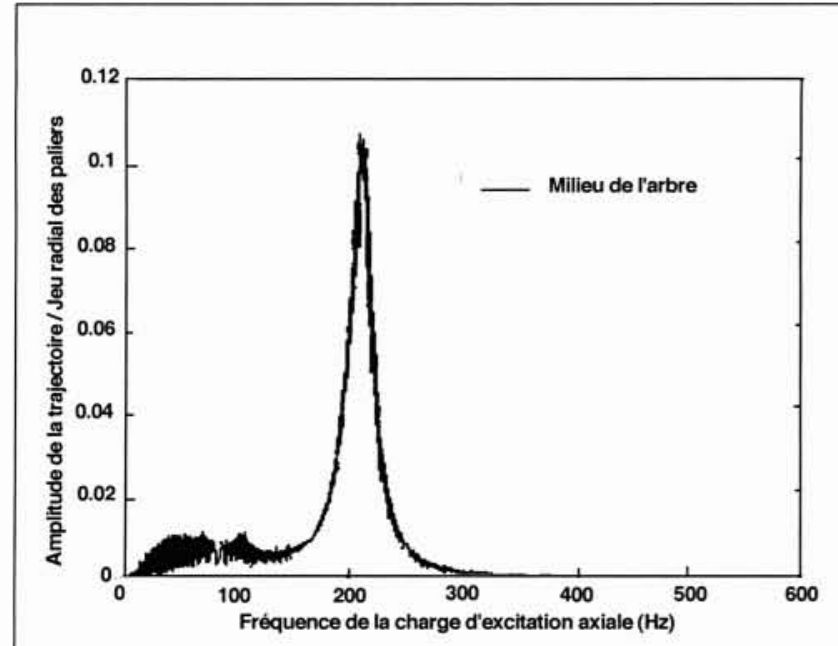

3. Amplitude des trajectoires du milieu de l'arbre.

\section{III — COMPORTEMENT GLOBAL DES LIGNES D'ARBRE}

Les ensembles tournants de turbomachines sont le plus souvent analysés à partir de deux types d'approches découplées. L'approche de type dynamique des rotors considère la flexibilité de l'arbre mais suppose que les roues qui sont montées dessus restent rigides et n'interviennent que par leurs masses et inerties [22]. L'approche de type disquesaubes quant à elle considère la flexibilité des roues mais suppose que l'arbre reste rigide. Ces deux types d'approches permettent de traiter efficacement une large gamme de problèmes couplés. La dynamique des rotors rend généralement bien compte des couplages rotors-paliers et l'approche disques-aubes est adaptée à l'étude de couplages courants : flottement des aubages ou effets de masse ajoutée par exemple. En revanche, il existe de plus en plus de configurations où la nécessité de considérer le comportement d'ensemble du système flexible arbre-roues-fluide apparaît clairement. Par exemple, les couplages déstabilisants liant les écoulements de fuite aux flasques de roues mettent en jeu la flexibilité de l'arbre, mais également selon toute vraisemblance la flexibilité des roues.

Pour avancer vers une analyse plus fine des interactions fluide-structure associées à la dynamique des rotors, un modèle spécifique a été développé et utilisé [23, 24]. Pour éviter des temps de calculs prohibitifs, ce modèle suppose que l'ensemble de la ligne d'arbre possède une symétrie cyclique d'ensemble (structures formée de secteurs identiques). Le problème couplé est projeté en base modale et résolu à l'aide d'un processus itératif. Le couplage entre les deux domaines, éléments finis pour la structure et différences finies pour le fluide, est réalisé à l'aide d'une grille d'interface. Dans une première phase d'analyse, seuls deux types de couplages ont été considérés : le couplage arbreroue et le couplage rotor-palier.

\subsection{Application}

L'application présentée concerne une roue de compresseur centrifuge expérimentale en acier comportant 13 aubes encadrées par deux flasques (Framatome Thermodyn). Le diamètre extérieur de la roue est de $0,685 \mathrm{~m}$, son épaisseur est

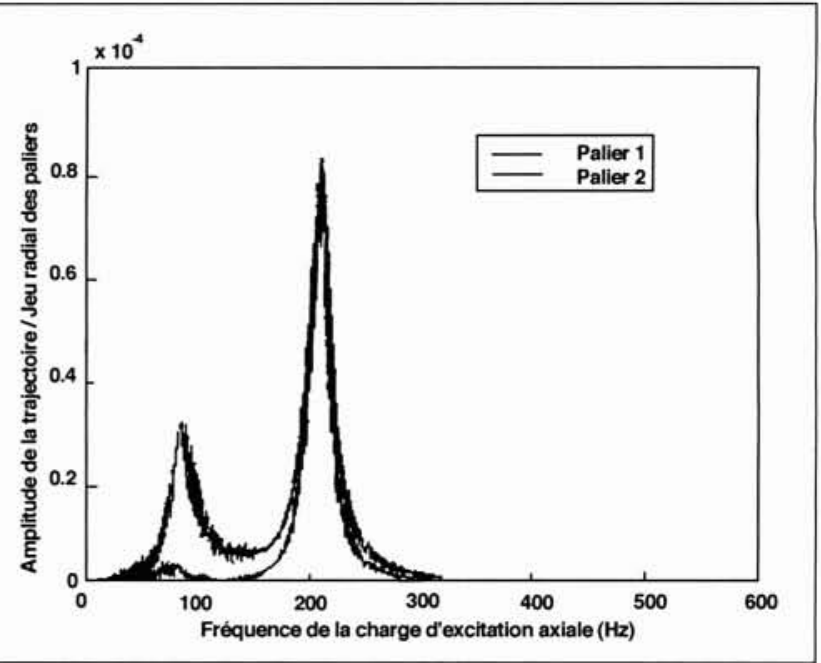

4. Amplitudes des trajectoires dans les paliers.

de $0,120 \mathrm{~m}$ et sa masse de $60,3 \mathrm{~kg}$. La roue est montée sur un arbre d'essai de longueur $0,5 \mathrm{~m}$ et de diamètre $0,06 \mathrm{~m}$ à chaque extrémité. Conformément au modèle utilisé, une portion de 1/13ème de l'ensemble roue-arbre est discrétisée à l'aide d'éléments finis isoparamétriques volumiques à 20 nœuds. Le maillage du secteur répétitif de base, présenté figure 5, comprend 178 éléments pour la roue et 91 éléments pour l'arbre.

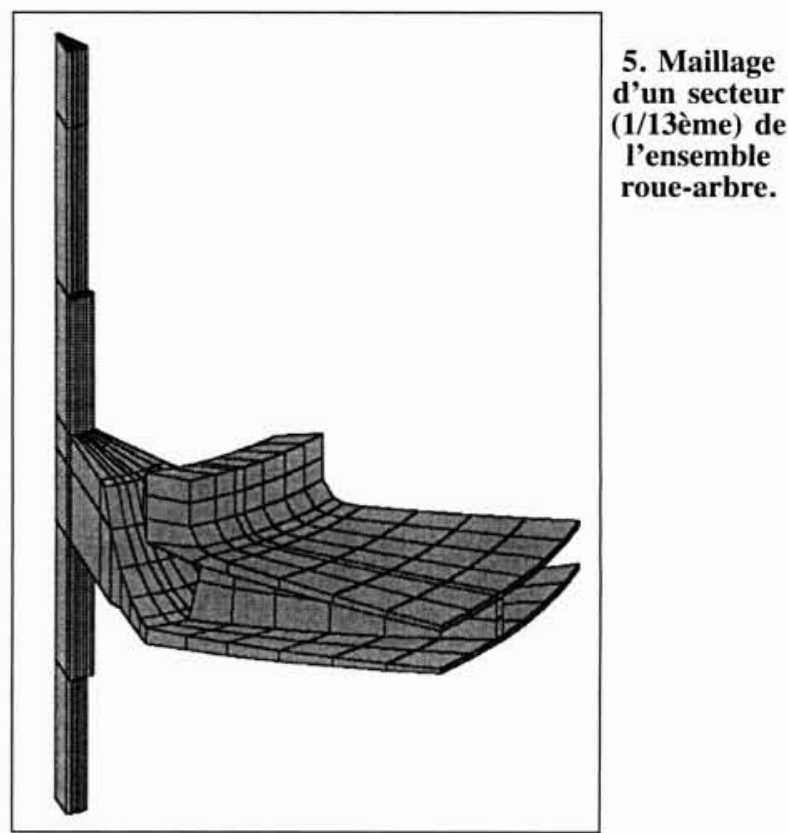

Dans un premier temps l'arbre est considéré en configuration simplement appuyé à ses deux extrémités, ce qui correspond par exemple à un montage sur roulements à billes. L'ensemble roue-arbre flexible est analysé et les résultats obtenus sont comparés avec ceux issus de modélisations classiques : roue rigide (dynamique des rotors) et arbre rigide (approche disques-aubes).

Les figures 6 et 7 présentent l'évolution des fréquences en fonction de la rotation (diagrammes de Campbell). La figure 6 concerne les premiers modes de flexion d'arbre et la figure 


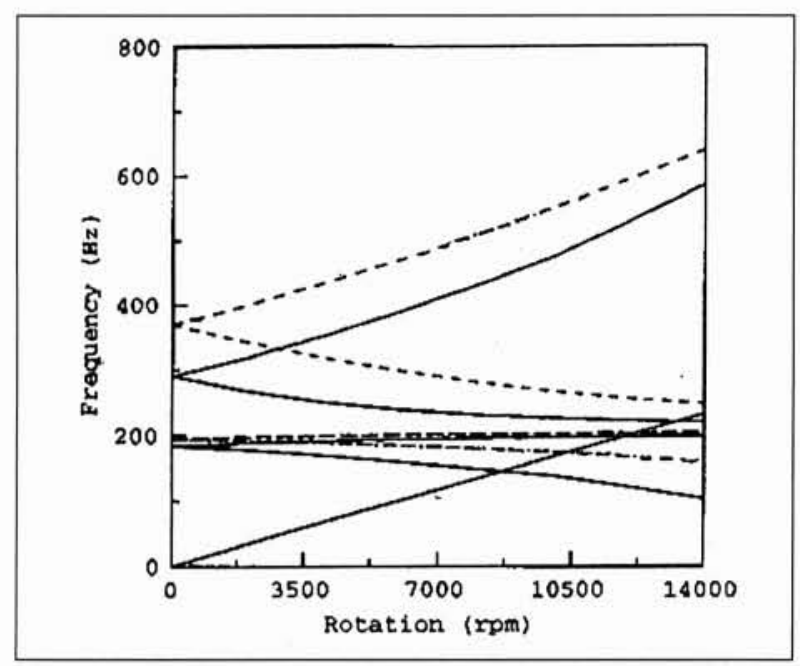

6. Modes d'arbre

- Roue flexible -.. Roue rigide.

7, une partie des modes de roues (un diamètre nodal). En examinant ces figures, on peut constater que sur la configuration spécifique considérée, l'influence des couplages rouearbre est très significative.

La structure est maintenant supposée maintenue rigidement à l'une des extrémités de l'arbre et montée sur un palier hydrodynamique lisse à l'autre extrémité. La longueur du palier est de $\mathrm{L}=0,024 \mathrm{~m}$, le jeu radial dans le palier est de $\mathrm{C}=10^{-4} \mathrm{~m}$ et la viscosité du fluide est $\mathrm{m}=0,01$ Pa.s. Une force verticale $\mathrm{F}=1000 \mathrm{~N}$ est appliquée sur le rotor au niveau du palier et un balourd de $50 \mathrm{~g} . \mathrm{cm}$ est imposé au niveau de la roue. La vitesse de rotation considérée, $11800 \mathrm{tr} / \mathrm{min}$, est proche de la première vitesse critique du rotor. Les équations relatives au palier sont résolues par discrétisation de type différences finies.

La comparaison des orbites obtenues au niveau du palier et au niveau de la roue avec les modèles à roue flexible $(\mathrm{F})$ et roue rigide (R), présentée figures 8 et 9 , montre ici aussi l'influence significative des couplages roue-arbre sur le comportement global du système.

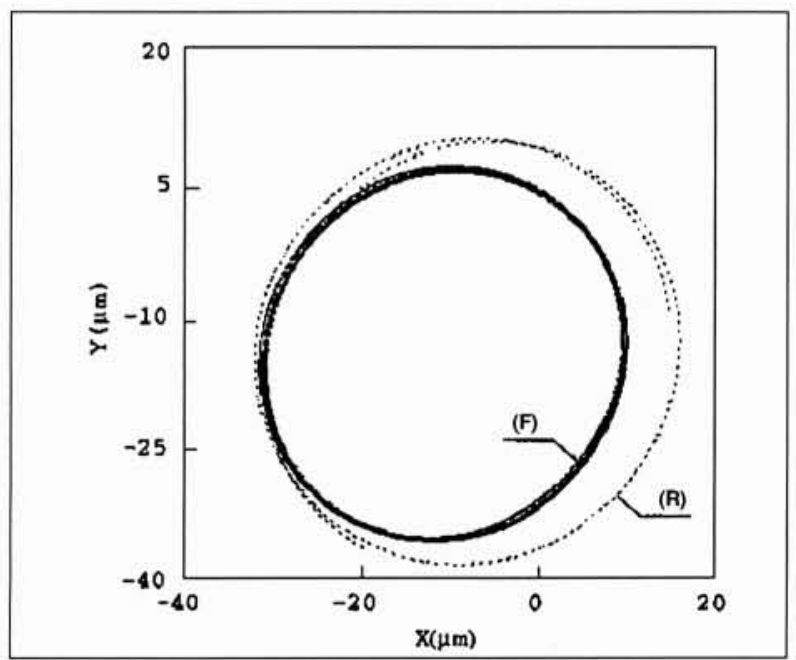

8. Orbite stabilisée au niveau du palier.

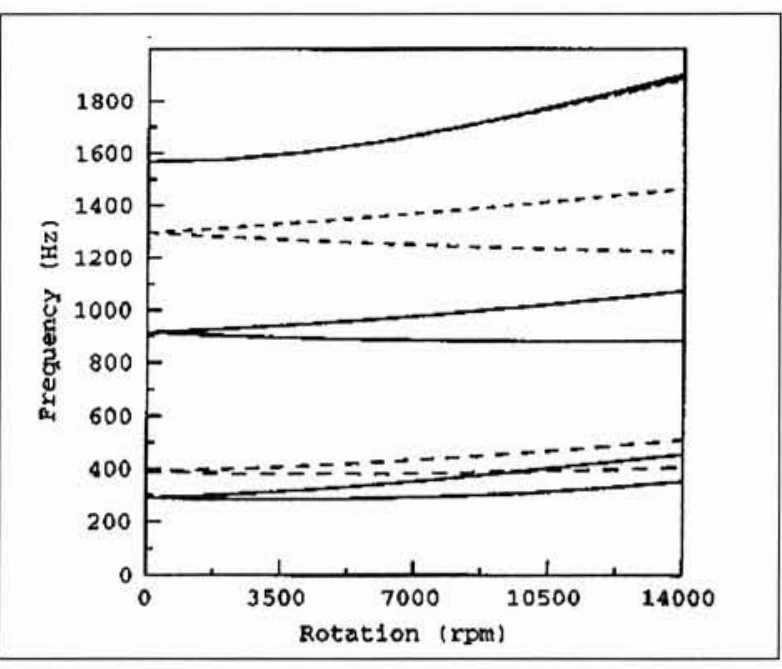

7. Modes de roue

- Arbre flexible -.. Arbre rigide.

\subsection{Conclusion}

L'analyse du comportement dynamique des ensembles tournants de turbomachines doit de plus en plus souvent être abordée de manière globale. En effet, il devient nécessaire de prendre en compte précisément les couplages possibles entre les différents sous-ensembles flexibles de la structure et entre la structure et le fluide, que ce soit le fluide véhiculé ou celui présent dans les paliers, dans les labyrinthes d'étanchéité, entre les flasques des roues et le carter par exemple. Quand les effets de la flexibilité des roues sont négligeables, l'approche classique de type dynamique des rotors est puissante et adaptée. En revanche, les limites de validité de l'hypothèse de roue rigide sont mal connues et les modèles susceptibles d'analyser le comportement global roues-arbre-fluide n'existent pas.

L'objectif de l'étude engagée est d'avancer vers l'établissement de tels modèles ainsi que vers une meilleure connaissance des phénomènes mis en jeu. Il s'agit, à terme, de pouvoir modéliser finement et d'analyser les interactions qui mettent directement en jeu les roues des lignes d'arbre.

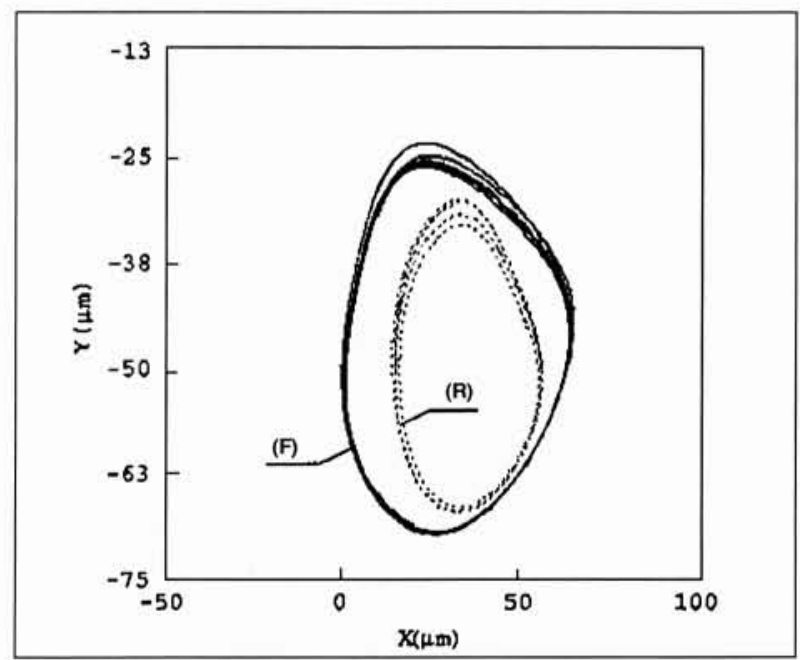

9. Orbite stabilisée au niveau de la roue. 


\section{BIBLIOGRAPHIE}

[1] SIMON F., FRENE J., "Analysis for Incompressible Flow in Annula Pressure Seals", ASME Journal of Tribology, vol. 114, 1991, pp.431438.

[2] LuCAS V., DANAila S., BONNEAU O., FRENE J., "Roughness Influence on Turbulent Flow Through Annular Seals", Journal of Tribology ASME, Vol. 116, April 1994, pp 321-329.

[3] LUCAS V, BONNEAU O., FRENE J., "Roughness Influence on the Turbulent Flow Through Annular Seals Including Inertia Effects", Journal of Tribology ASME, Vol. 118, January 1996, pp 175-182

[4] JACQUET-RICHARDET G., DAL-FERRO C., "Reduction method for finite element dynamic analysis of submerged turbomachinery wheels", Computers and structures, vol. 61 (6), 1996, pp 1025-1036.

[5] JACQUET-RICHARDET G., "Comportement dynamique des ensembles tournants de turbomachines couplés aux fluides environnants", La Houille Blanche, vol. 3/4, 1998, pp. 93-98.

[6] JACQUET-RICHARDET G., MOYROUD F., FRANSSON T.H., "An influence of shroud design on the dynamic and aeroelastic behavior of bladed disc assemblies". ASME Gas Turbine and Aeroengine Congress, ASME Paper 97GT191, 1997

[7] MOYROUD F., JACQUET-RICHARDET G., FRANSSON T.H., "Aeroelasticity in turbomachines: effect of coupling modeling and blade material changes", Proceedings of the ISROMAC-7, Honolulu, 1998. To be published in the International Journal of Rotating Machinery.

[8] PECHEUX B., BONNEAU O., FRENE J., "Investigation about Electro-Rheological Squeeze Film Damper Applied to Active Control of Rotor Dynamic", International Journal of Rotating Machinery, Vol. 3. issue 1, January 1997.

[9] BONNEAU O., FRENE J. "Numerical study of a flexible rotor mounted in an active squeeze film damper", Fourth International Conference on Rotor Dynamics, IFToMM 1994 Chicago pp 327-331.

[10] ABDUL-WAHED N. "Comportement dynamique des paliers fluides. Etude linéaire et non linéaire". Thèse de doctorat d'état, Université Claude Bernard, Lyon, 1982.

[11] BONNEAU O "Comportement Statique et Dynamique de ligne d'Arbre Montée sur Paliers Fluides : Influence des Caractéristiques des Paliers", Thèse de I'Université de Poitiers, février 1989.

[12] BONNEAU O., KASSAI A., FRENE J., DER HAGOPIAN J., "Dyna- mical behavior of an elastic rotor with squeeze film damper" EUROTRIB HELSINKI FINLANDE juin 89 Proceeding Vol.4, pp 145-149.

[13] PERSON V., TOURNERIE B., FRENE J., "A numerical study of the stable dynamic behavior of radial face seals with grooved faces", ASME/STLE International Tribology Conference, ASME 96 - TRIB40, San Francisco, 13-17 octobre 1996.

[14] CICONE T., TOURNERIE B., REUNGOAT D., DANOS J.C., "Startup transient temperature distribution in a mechanical face seal", World Tribology Congress, Londres, 8-12 Septembre 1997

[15] PERSON V., TOURNERIE B., FRENE J., "THD aspects in aligned face seals", World Tribology Congress, Londres, 8-12 Septembre 1997

[16] PERSON V., TOURNERIE B., "THD aspects in misaligned wavy face seals", 15th. International Conference on Fluid Sealing, BHRGroup. Maastricht, September 1997.

[17] Arghir, M., Frêne, J, "Analysis Of A Test Case For Annular Seal Flows", Transactions of the ASME Journal of Tribology, Vol. 119, pp. 408-415.

[18] Arghir, M., Frêne, J, "Rotordynamic Coefficients of CircumferentiallyGrooved Liquid Seals Using the Averaged Navier-Stokes Equations", Transactions of the ASME Journal of Tribology, Vol. 119, pp. 556-567.

[19] KASSAI A., 1989 "Contribution à l'Etude Dynamique des Rotors Amortis", Thèse de Doctorat de l'INSA de Lyon, juillet 1989

[20] BERGER S., Etude du comportement dynamique non linéaire d'un rotor monté sur éléments fluides-Influence des étanchéités - Rôle de la butée, Thèse de l'Université de Poitiers, 1999.

[21] BERGER S., BONNEAU O., FRENE J. "Influence of Axial Thrust Bearing Defects on the Dynamic Behavior of an Elastic Shaft" AUS. TRIB 98 Conference, Brisbane 6-9 décembre 1998, actes du congrès.

[22] LALANNE M., FERRARIS G., "Rotordynamics prediction in Engineering", 2nd Edition, J. Wiley, Chichester, 1997

[23] JACQUET-RICHARDET, G., FERRARIS G., RIEUTORD P., "Frequencies and modes of rotating flexible bladed disc-shaft assemblies : A global cyclic symmetry approach". Journal of Sound and Vibration vol 191 (5), 1996, pp 901-915

[24] JACQUET-RICHARDET G., RIEUTORD P., "A three dimensional fluidstructure coupled analysis of rotating flexible assemblies of turbomachines", Journal of Sound and Vibration, vol. 209 (1), 1998, pp. 61-76. 